LANDAU-92-TMP-2

December 1992

Submitted to Mod. Phys. Lett. A

\title{
SUPERCONFORMAL 2D MINIMAL MODELS AND AN UNUSUAL COSET CONSTRUCTION
}

\author{
M. YU. LASHKEVICH* \\ Landau Institute for Theoretical Physics, Academy of Sciences, \\ Kosygina 2, GSP-1, 117940 Moscow V-334, Russian Federation **
}

We consider a coset construction of minimal models. We define it rigorously and prove that it gives superconformal minimal models. This construction allows to build all primary fields of superconformal models and to calculate their three-point correlation functions.

Superconformal two-dimensional unitary minimal models ${ }^{1-4}$ are theories with chiral algebra which is generated by energy-momentum tensor $T^{S}(z)$ of spin 2 and its superpartner $J(z)$ of spin $\frac{3}{2}$. Their operator product expansions are given by

$$
\begin{aligned}
& T^{S}\left(z^{\prime}\right) T^{S}(z)=\frac{\frac{1}{2} c^{S}}{\left(z^{\prime}-z\right)^{4}}+\frac{2 T^{S}(z)}{\left(z^{\prime}-z\right)^{2}}+\frac{\partial T^{S}(z)}{z^{\prime}-z}+O(1), \\
& T^{S}\left(z^{\prime}\right) J(z)=\frac{\frac{3}{2} J(z)}{\left(z^{\prime}-z\right)^{2}}+\frac{\partial J(z)}{z^{\prime}-z}+O(1), \\
& J\left(z^{\prime}\right) J(z)=\frac{\frac{3}{2} c^{S}}{\left(z^{\prime}-z\right)^{3}}+\frac{2 T^{S}(z)}{z^{\prime}-z}+\partial T^{S}(z)+O\left(z^{\prime}-z\right),
\end{aligned}
$$

with $\partial \equiv \partial / \partial z$, and $c^{S}$ be a central charge of the Virasoro algebra $(1 \mathrm{a})^{\mathrm{a}}$

$$
c_{k}^{S}=\frac{3}{2}-\frac{12}{(k+2)(k+4)} ; k=1,2,3, \cdots .
$$

We will designate these models as $\mathrm{SM}_{k}$. It is known ${ }^{5}$ that superconformal minimal models can be obtained as a result of coset construction of SU(2) Wess-Zumino models:

$$
\mathrm{SM}_{k} \sim \frac{\mathrm{SU}(2)_{k} \times \mathrm{SU}(2)_{2}}{\mathrm{SU}(2)_{k+2}}
$$

* This work was supported, in part, by Landau Scolarship Grant awarded by Forschungszentrum Jülich GmbH, and by Soros Foundation Grant awarded by the American Physical Society

** E-mail: ngm@cpuv1.net.kiae.su with note 'Lashkevich/Landau Inst.' in Subject.

a We use the definition of central charge common for conformal models. It is $\frac{3}{2}$ of superconformal one. 
Index at $\mathrm{SU}(2)$ designates central charge of Kac-Moody algebra. ${ }^{6}$ On the other hand, conformal minimal model $\mathrm{M}_{k}$ with central charge

$$
c_{k}=1-\frac{6}{(k+2)(k+3)}
$$

can be obtained by coset construction too

$$
\mathrm{M}_{k} \sim \frac{\mathrm{SU}(2)_{k} \times \mathrm{SU}(2)_{1}}{\mathrm{SU}(2)_{k+1}}
$$

Let us make a naive transformation

$$
\begin{aligned}
\mathrm{SM}_{k} & \sim \frac{\mathrm{SU}(2)_{k} \times \mathrm{SU}(2)_{1}}{\mathrm{SU}(2)_{k+1}} \times \frac{\mathrm{SU}(2)_{k+1} \times \mathrm{SU}(2)_{1}}{\mathrm{SU}(2)_{k+2}} \times \frac{\mathrm{SU}(2)_{2}}{\mathrm{SU}(2)_{1} \times \mathrm{SU}(2)_{1}} \\
& \sim \frac{\mathrm{M}_{k} \times \mathrm{M}_{k+1}}{\mathrm{M}_{1}} .
\end{aligned}
$$

The last expression is quite unusual. Can we assign an exact meaning to it? Before we make it we mention a generalization to coset constructions

$$
\begin{gathered}
\mathrm{N}_{k l} \sim \frac{\mathrm{SU}(2)_{k} \times \mathrm{SU}(2)_{l}}{\mathrm{SU}(2)_{k+l}} \text {.Inthesamewayasin(6)wecanwrite } \\
\mathrm{N}_{k l} \sim \frac{\mathrm{N}_{k, l-m} \times \mathrm{N}_{k+l-m, m}}{\mathrm{~N}_{l-m, m}},
\end{gathered}
$$

in particular

$$
\mathrm{N}_{k l} \sim \frac{\mathrm{N}_{k, l-1} \times \mathrm{M}_{k+l-1}}{\mathrm{M}_{l-1}} \sim \frac{\mathrm{M}_{k} \times \mathrm{N}_{k+1, l-1}}{\mathrm{M}_{l-1}}
$$

We defer consideration of this general case to a more detailed paper.

Now we will look at the representation of minimal model $\mathrm{M}_{k}$ by one bosonic field $\varphi(z)^{7-10}$ with correlation function

$$
\left\langle\varphi\left(z^{\prime}\right) \varphi(z)\right\rangle=-\ln \left(z^{\prime}-z\right)
$$

and energy-momentum tensor

$$
T_{k}(z)=-\frac{1}{2}:(\partial \varphi)^{2}:+\frac{i}{\sqrt{2(k+2)(k+3)}} \partial^{2} \varphi
$$

colons mean normal ordering. Vertex operators, $V_{(p, q)}^{(k) m n}(z)$, with conformal dimensions

$$
\Delta_{(p, q)}^{(k)}=\frac{[(k+3) p-(k+2) q]^{2}-1}{4(k+2)(k+3)}
$$


are given by

$$
\begin{gathered}
\underset{V_{(p, q)}^{(k) m n}(z)}{ }=V_{(p, q)}^{(k)}(z) \prod_{i=1}^{m} \oint_{C_{i}} d u_{i} I_{+}^{(k)}\left(u_{i}\right) \prod_{i=1}^{n} \oint_{C_{i}} d v_{i} I_{-}^{(k)}\left(v_{i}\right), \\
V_{(p, q)}^{(k)}(z)=: \exp \left(-i\left(\frac{p-1}{2} \sqrt{2 \frac{k+3}{k+2}}-\frac{q-1}{2} \sqrt{2 \frac{k+2}{k+3}}\right) \varphi(z)\right): \\
p=1, \cdots, k+1 ; \quad q=1, \cdots, k+2
\end{gathered}
$$

where screenings,

$$
\begin{aligned}
& I_{+}^{(k)}(z)=: \exp \left(i \sqrt{2 \frac{k+3}{k+2}} \varphi\right): \\
& I_{-}^{(k)}(z)=: \exp \left(-i \sqrt{2 \frac{k+2}{k+3}} \varphi\right):
\end{aligned}
$$

are fields with conformal dimension 1; contours of integration are presented in Fig. 1. Recall that vertex operators form an irreducible representation for quantum $\operatorname{group}^{11} \mathrm{SL}_{q(k)}(2) \odot \mathrm{SL} \frac{}{q(k+1)}(2), q(k)=\exp \left(\frac{2 \pi i}{k+2}\right)$; bar means complex conjugation; sign $\odot$ means a special kind of product. ${ }^{11,12} \mathrm{~b}$ This representation have 'full momenta' $J_{1}=\frac{1}{2}(p-1), J_{2}=\frac{1}{2}(q-1)$ for two $\mathrm{SL}(2)$ groups. 'Projections of momenta' are $M_{1}=J_{1}-m, M_{2}=J_{2}-n$.

From two representations with equal dimensions, the first for group $\mathrm{SL}_{q}(2)$ and the second for $\mathrm{SL}_{\bar{q}}(2)$, a bilinear braiding ( $R$-matrix) invariant can be constructed. Therefore, invariant primary field can be constructed as

$$
\phi_{(p, q)}^{(k)}(z, \bar{z})=N_{(p, q)} \sum_{m=0}^{p-1} \sum_{n=0}^{q_{1}} X_{p}^{(k)}(m) X_{q}^{(k+1)}(n) V_{(p, q)}^{(k) m n}(z) V_{(p, q)}^{(k) m n}(\bar{z})
$$

where $X_{p}^{(k)}(m)$ are the coefficients of invariants for $\mathrm{SL}_{q(k)}(2)$ quantum group, and $N_{(p, q)}$ is a normalization factor which will be omitted later. Notice that this braiding invariance provides monodromy invariance ${ }^{7,8}$ or, equivalently, duality ${ }^{13}$ of correlation functions.

Let us construct a vertex operator

$$
W_{(p, s, q)}^{m n}(z)=\sum_{r=0}^{s-1} X_{s}^{(k+1)}(r) V_{(p, s)}^{(k) m r}(z) V_{(s, q)}^{(k+1) r n}(z)
$$

in the theory $\mathrm{M}_{k} \times \mathrm{M}_{k+1}$. This vertex realizes a represetation of the quantum group $\mathrm{SL}_{q(k)}(2) \times \mathrm{SL} \frac{}{q(k+2)}(2)$. We have constructed the numerator of the coset $(6)$. Now we will build the denominator.

b The origin of this product is in hidden $\mathrm{SL}_{q(1)}(2)$ group which corresponds to the monodromy trivial situation. Full quantum group of minimal model $\mathrm{M}_{k}$ is ${ }^{15}$ $\mathrm{SL}_{q(k)}(2) \times \mathrm{SL}_{q(1)}(2) \times \mathrm{SL} \frac{}{q(k+1)}(2)$ [see also Eq. (5)]. 
The superconformal minimal model $\mathrm{SM}_{k}$ possesses a representation by three bosons $^{14} \chi(z), \rho(z)$ and $\Phi(z)$ with non-zero correlation functions

$$
\left\langle\chi\left(z^{\prime}\right) \chi(z)\right\rangle=\left\langle\rho\left(z^{\prime}\right) \rho(z)\right\rangle=\left\langle\Phi\left(z^{\prime}\right) \Phi(z)\right\rangle=-\ln \left(z^{\prime}-z\right),
$$

and energy-momentum tensor

$$
\begin{aligned}
T_{k}^{S}(z)=-\frac{1}{2}(\partial \chi)^{2}-\frac{i}{2} \partial^{2} \chi & -\frac{1}{2}(\partial \rho)^{2}+\frac{1}{2 \sqrt{2}} \partial^{2} \rho \\
& -\frac{1}{2}(\partial \Phi)^{2}+\frac{i}{\sqrt{(k+2)(k+4)}} \partial^{2} \Phi .
\end{aligned}
$$

The supercurrent is given by (up to a normalization factor)

$$
\begin{aligned}
& J(z) \sim \oint_{C_{z}} \frac{d u}{2 \pi i}: \exp (i(k+1) \chi-(k+2) \sqrt{2} \rho-i \sqrt{(k+2)(k+4)} \Phi):(u) \\
& \cdot: \exp \left(-i k \chi+\frac{2 k+3}{\sqrt{2}} \rho+i \sqrt{(k+2)(k+4)} \Phi\right):(z) .
\end{aligned}
$$

Vertex operators are given by

$$
\begin{aligned}
& S_{p p^{\prime} q}^{(k) m m^{\prime} n}(z)=S_{p p^{\prime} q}^{(k)}(z) \\
& \cdot \prod_{i=1}^{m} \oint_{C_{i}} d u_{i} I_{1}\left(u_{i}\right) \prod_{i=1}^{m^{\prime}} \oint_{C_{i}^{\prime}} d v_{i} I_{1}^{\prime}\left(v_{i}\right) \prod_{i=1}^{n} \oint_{S_{i}} d w_{i} I_{2}\left(w_{i}\right), \\
& S_{p p^{\prime} q}^{(k)}=f_{p+p^{\prime}-q-1+4 N}(z): \exp \left\{\frac{2(q-p-4 N)-\left(p^{\prime}-1\right)}{2 \sqrt{2}} \rho\right. \\
& \left.-i \frac{(k+4)(p-1)-(k+2)(q-1)}{2 \sqrt{(k+2)(k+4)}} \Phi\right\}:(z), \\
& p=1, \cdots, k+1 ; \quad p^{\prime}=1,2,3 ; \quad q=1, \cdots, k+3 ; \\
& p+p^{\prime}-q-1 \in 2 \mathbf{Z}, \quad(1,3,1) \neq\left(p, p^{\prime}, q\right) \neq(k+1,1, k+4), \\
& 0 \leq q+p^{\prime}-p-1-4 N<4, \quad N \in \mathbf{Z} \text {, } \\
& f_{2 n}(z)= \begin{cases}: e^{i n \chi}:(z) & \text { if } n \geq 0, \\
\oint_{C_{z}} \frac{d u}{2 \pi i}: e^{i \chi}:(u): e^{-i(1-n) \chi}:(z) & \text { if } n \leq 0,\end{cases}
\end{aligned}
$$

contours $C_{i}, C_{i}^{\prime}$ and $S_{i}$ are as in Fig. 1 , contour $C_{z}$ is a small circle around $z$. Screening fields are given by ${ }^{\mathrm{c}}$

$$
\begin{aligned}
& I_{1}(z)=\oint_{C_{z}} \frac{d u}{2 \pi i}: e^{3 i \chi-2 \sqrt{2} \rho}:(u): \exp \left(-2 i \chi+\sqrt{2} \rho+i \sqrt{\frac{k+4}{k+2}} \Phi\right):(z), \\
& I_{1}^{\prime}(z)=\oint_{C_{z}} \frac{d u}{2 \pi i}: e^{i \chi}:(u): \exp \left(-2 i \chi+\frac{1}{\sqrt{2}} \rho\right):(z), \\
& I_{2}(z)=\oint_{C_{z}} \frac{d u}{2 \pi i}: e^{i \chi}:(u): \exp \left(-2 i \chi+\sqrt{2} \rho-i \sqrt{\frac{k+2}{k+4}} \Phi\right):(z) .
\end{aligned}
$$

${ }^{\mathrm{c}}$ In Ref. 14 they are designated as $I_{1} \tilde{Z}, I_{1}^{\prime}$ and $I_{2} Z$ correspondingly. 
Note that the supercurrent $J(z)$ does not commute with the screening operator $\oint d u I_{1}^{\prime}(u)$. It can be proved, however, that $J(z)$ exhibits trivial monodromy properties. ${ }^{15}$ Indeed, using Dotsenko procedure ${ }^{16}$ we obtain immidiately the fusion rule

$$
J\left(z^{\prime}\right) \psi_{p p^{\prime} q}^{(k)}(z) \sim\left(z^{\prime}-z\right)^{-3 / 2 \pm 1 / 2}\left[\psi_{p, 4-p^{\prime}, q}^{(k)}\right]
$$

where $\psi_{p p^{\prime} q}^{(k)}$ is the field described by vertex $S_{p p^{\prime} q}^{(k) m m^{\prime} n}(z)$, brackets mean the field with its entire Fock module. This means that, if $z^{\prime}$ goes around $z$ and returns to the initial point, the product only changes by a constant factor (here unity), and any correlation function with chiral (holomorphic) current $J(z)$ is well defined.

Conformal dimensions of vertices (10) are given by ${ }^{2-4,14}$

$$
\Delta_{p p^{\prime} q}^{(k)}=\frac{[(k+4) p-(k+2) q]^{2}-4}{8(k+2)(k+4)}
$$

if $p-q \in 2 \mathbf{Z}, p^{\prime}-1=p-q(\bmod 4)$ (Neveu-Schwarz primaries),

$$
\Delta_{p p^{\prime} q}^{(k)}=\frac{[(k+4) p-(k+2) q]^{2}-4}{8(k+2)(k+4)}+\frac{1}{2},
$$

if $p-q \in 2 \mathbf{Z}, p^{\prime}-3=p-q(\bmod 4)$ (Neveu-Schwarz superpartners),

$$
\Delta_{p p^{\prime} q}^{(k)}=\frac{[(k+4) p-(k+2) q]^{2}-4}{8(k+2)(k+4)}+\frac{1}{16},
$$

if $p-q \in 2 \mathbf{Z}+1$ (Ramond primaries).

Quantum group of the superconformal minimal model is $\mathrm{SL}_{q(k)}(2) \times \mathrm{SL}_{q(2)}(2)$ $\times \mathrm{SL} \frac{}{q(k+2)}(2)$, and invariant primary fields are

$$
\psi_{p p^{\prime} q}^{(k)}(z, \bar{z})=\sum_{m m^{\prime} n} X_{p}^{(k)}(m) X_{p^{\prime}}^{(2)}\left(m^{\prime}\right) X_{q}^{(k+2)}(n) S_{p p^{\prime} q}^{(k) m m^{\prime} n}(z) S_{p p^{\prime} q}^{(k) m m^{\prime} n}(\bar{z}) .
$$

In Neveu-Schwarz case monodromy invariant fields can be obtained without summation over $m^{\prime}$ by the same reason as $J(z)$ is a chiral current [see Eq. (12)], and the following expression is already monodromy invariant:

$$
\psi_{p p^{\prime} p^{\prime \prime} q}^{(k) \prime}(z, \bar{z})=\sum_{m n} X_{p}^{(k)}(m) X_{q}^{(k+2)}(n) S_{p p^{\prime} q}^{(k) m n}(z) S_{p p^{\prime \prime} q}^{(k) m n}(\bar{z}) .
$$

Here absence of $m^{\prime}$ in vertex means that necessary summation is already done in each vertex separately.

Consider now a vertex in $\mathrm{M}_{2} \times \mathrm{SM}_{k}$ theory

$$
U_{p p^{\prime} q, t}^{m n}(z)=\sum_{m^{\prime}=1}^{p^{\prime}-1} X_{p^{\prime}}^{(2)}\left(m^{\prime}\right) V_{\left(t, p^{\prime}\right)}^{(1) r m^{\prime}}(z) S_{p p^{\prime} q}^{(k) m m^{\prime} n}(z), \quad t=1,2 .
$$


Index $r$ corresponds to $\mathrm{SL}_{q(1)}(2)$ quantum group which describes monodromy trivial situation, and we omit it in the l.h.s. Let us prove that the theory generated by vertices $W_{(p, s, q)}^{m n}(z)$ from Eq. (9) contains the theory generated by vertices $U_{p p^{\prime} q, t}^{m n}(z)$. We will apply the method used in the proof of equivalence of coset theory $\mathrm{SU}(2)_{k} \times \mathrm{SU}(2)_{l} / \mathrm{SU}(2)_{k+l}$ and minimal-like bosonic models. ${ }^{15}$ Namely, we construct chiral currents $T_{k}(z)$ and $T_{k+1}(z)$ of $\mathrm{M}_{k} \times \mathrm{M}_{k+1}$ theory from vertices (14) of $\mathrm{M}_{1} \times \mathrm{SM}_{k}$ theory:

$$
\begin{gathered}
T_{k}(z)=\frac{1}{2} \frac{k}{k+3} T_{1}(z)+\frac{1}{2} \frac{k+4}{k+3} T_{k}^{S}(z)+\frac{\sqrt{(k+2)(k+4)}}{2(k+3)} J(z) \phi_{(2,1)}^{(1) \prime}(z) \\
T_{k+1}(z)=\frac{1}{2} \frac{k+6}{k+3} T_{1}(z)+\frac{1}{2} \frac{k+2}{k+3} T_{k}^{S}(z)-\frac{\sqrt{(k+2)(k+4)}}{2(k+3)} J(z) \phi_{(2,1)}^{(1) \prime}(z) \\
T_{k}(z)+T_{k+1}(z)=T_{1}(z)+T_{k}^{S}(z)
\end{gathered}
$$

where $\phi_{(2,1)}^{(1) \prime}(z)$ is a chiral field in $\mathrm{M}_{1} . \mathrm{M}_{1}$ with such chiral field is known to coinside with fermion theory. ${ }^{1,13,17}$ The field $\phi_{(2,1)}^{(1) \prime}(z)$ is the fermion current with spin $\frac{1}{2}$ and operator product expansion

$\phi_{(2,1)}^{(1) \prime}\left(z^{\prime}\right) \phi_{(2,1)}^{(1) \prime}(z)=\left(z^{\prime}-z\right)^{-1}+2\left(z^{\prime}-z\right) T_{1}(z)+\left(z^{\prime}-z\right)^{2} \partial T_{1}(z)+O\left(\left(z^{\prime}-z\right)^{3}\right)$.

Correct operator product expansions for $T_{k}(z)$ and $T_{k+1}(z)$ from Eq. (26) are directly obtained from Eqs. (1) and (17). These energy-momentum tensors act locally on vertices $U_{p p^{\prime}, t}^{m n}(z)$, and therefore, these vertices belong to $\mathrm{M}_{k} \times \mathrm{M}_{k+1}$. Quantum group properties show us that these vertices belong to the subtheory generated by vertices $W_{(p, s, q)}^{m n}(z)$.

Note that one can uniquely determine conformal blocks of $S_{p p^{\prime} q}^{(k) m m^{\prime} n}(z)$ if those of $U_{p p^{\prime} q, t}^{m n}(z)$ and $V_{\left(t, p^{\prime}\right)}^{(2) r m^{\prime}}(z)$ are known. ${ }^{18}$ Indeed, matrix $V_{i}^{j}=\left\langle V_{\left(t, p^{\prime}\right)}^{(2) r m^{\prime}} \cdots\right\rangle$, $i=\left(t, p^{\prime}, \ldots\right), j=(r, m, \ldots)$, truncated in evident way is indegenerate, because its determinant is Wronskian of linearly independent solutions of those differential equations which define conformal theory $\mathrm{M}_{1}$.

Let us write out $T_{1}(z), T_{k}^{S}(z)$, and $J(z)$ through $\mathrm{M}_{k} \times \mathrm{M}_{k+1}$ fields. Consider the field

$$
t(z)=W_{(1,3,1)}(z)
$$

Its monodromy properties are trivial and its operator product expansion can be calculated using bosonic representations for $\mathrm{M}_{k}$ and $\mathrm{M}_{k+1}$ in just the same way as tree-point correlation functions for minimal models. ${ }^{8,9}$ Instead of gluing together holomorphic and antiholomorphic parts in calculation of correlation functions we 
must glue together $\mathrm{M}_{k}$ and $\mathrm{M}_{k+1}$ parts of the field. The result is

$$
\begin{array}{r}
t\left(z^{\prime}\right) t(z)=\frac{1}{\left(z^{\prime}-z\right)^{4}}+\frac{2 \theta(z)}{\left(z^{\prime}-z\right)^{2}}+\frac{\partial \theta(z)}{z^{\prime}-z}+O(1) \\
\theta(z)=\frac{2(k-1)(k+2)}{\sqrt{3 k(k+1)(k+5)(k+6)}} t(z)+\frac{(k+1)(k+2)}{k(k+5)} T_{k}(z) \\
+\frac{(k+4)(k+5)}{(k+1)(k+6)} T_{k+1}(z) .
\end{array}
$$

Finally, we have

$$
\begin{aligned}
T_{1}(z) & =\frac{1}{4} \frac{k+2}{k+5} T_{k}(z)+\frac{1}{4} \frac{k+4}{k+1} T_{k+1}(z)+\frac{1}{4} \sqrt{3 \frac{k(k+6)}{(k+1)(k+5)}} t(z) \\
T_{k}^{S}(z)=\frac{3}{4} \frac{k+6}{k+5} T_{k}(z)+\frac{3}{4} \frac{k}{k+1} T_{k+1}(z) & -\frac{1}{4} \sqrt{3 \frac{k(k+6)}{(k+1)(k+5)}} t(z), \\
\phi_{(2,1)}^{(1) \prime}(z) J(z)=\sqrt{3 \frac{k(k+6)}{(k+1)(k+2)(k+3)(k+5)}} t(z) & +\frac{1}{2} \frac{(k+2)(k+6)}{(k+3)(k+5)} T_{k}(z) \\
& -\frac{1}{2} \frac{k(k+4)}{(k+1)(k+3)} T_{k+1}(z) .
\end{aligned}
$$

Now we can make field identification. Consider operator product expansion

$$
\begin{aligned}
t\left(z^{\prime}\right) W_{(p, s, q)}(z) \sim\left(z^{\prime}-z\right)^{-2}\left[W_{(p, s, q)}\right] & +\left(z^{\prime}-z\right)^{-2 s+p+q}\left[W_{(p, s-2, q)}\right] \\
& +\left(z^{\prime}-z\right)^{2 s-p-q}\left[W_{(p, s+2, q)}\right] .
\end{aligned}
$$

Fields $W_{(p, s \pm 2, q)}$ do not give poles of the order $>2$ if

$$
\frac{1}{2}(p+q)-1 \leq s \leq \frac{1}{2}(p+q)+1
$$

This means that, if the condition $(22)$ is satisfied, the fields $W_{(p, s, q)}(z)$ can be primary. We will discuss all cases in sequence.

1. $p+q \in 2 \mathbf{Z}, s=\frac{1}{2}(p+q)$. Considering operator product expansions of

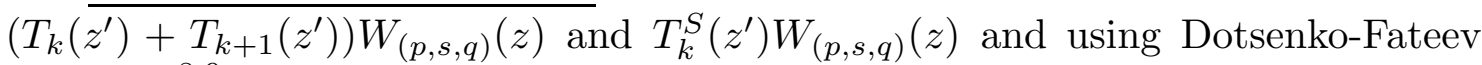
technique $^{8,9}$ we calculate conformal dimensions and see that we have NeveuSchwarz primary fields

$$
S_{p p^{\prime} q}^{(k) m n}(z)=W_{(p, s, q)}^{m n}(z), \quad s=\frac{1}{2}(p+q), \quad p^{\prime}-1=p-q(\bmod 4) .
$$

2. $p+q \in 2 \mathbf{Z}+1, s=\frac{1}{2}(p+q \pm 1)$. In a similar manner we obtain Ramond primaries

$$
\sum_{m^{\prime}=0}^{1} X_{2}^{(2)}\left(m^{\prime}\right) V_{(1,2)}^{(1) 0, m^{\prime}}(z) S_{p, 2, q}^{(k) m n}(z)=W_{(p, s, q)}^{m n}(z), \quad s=\frac{1}{2}(p+q \pm 1) .
$$


3. $p+q \in 2 \mathbf{Z}, s_{ \pm}=\frac{1}{2}(p+q) \pm 1$. This case is a bit more complex, because

$$
\Delta_{\left(p, s_{-}, q\right)}=\Delta_{\left(p, s_{+}, q\right)},
$$

and action of $L_{0}^{S}=\oint_{C_{z}} \frac{d u}{2 \pi i} u T_{k}^{S}(u)$ mixes $\phi_{\left(p, s_{-}, q\right)}$ and $\phi_{\left(p, s_{+}, q\right)}$. Finding eigenvalues and eigenvectors of the 'Hamiltonian matrix' we obtain two kinds of fields: Neveu-Schwarz primaries

$$
\begin{aligned}
\phi_{(2,1)}^{(1) \prime}(z) S_{p p^{\prime} q}^{(k) m n}(z) & =\sqrt{\frac{1}{2}+y} W_{\left(p, s_{+}, q\right)}^{m n}(z)-\sqrt{\frac{1}{2}-y} W_{\left(p, s_{-}, q\right)}^{m n}(z), \\
y & =[(k+4) p-(k+2) q]^{-1}
\end{aligned}
$$

and Neveu-Schwarz superpartners

$$
\begin{gathered}
S_{p p^{\prime} q}^{(k) m n}(z)=\sqrt{\frac{1}{2}-y} W_{\left(p, s_{+}, q\right)}^{m n}(z)+\sqrt{\frac{1}{2}+y} W_{\left(p, s_{-}, q\right)}^{m n}(z), \\
s_{ \pm}=\frac{1}{2}(p+q) \pm 1, \quad p^{\prime}-3=p-q(\bmod 4) .
\end{gathered}
$$

Eqs. (23-26) allow to calculate structure constants $C_{a b c}^{S(k)}$, i.e. coefficients in tree-point correlation functions of invariant primary fields $a, b$ and $c$. Indeed, it is easy to check that, if we glue together two vertex operators in the abovementionned manner, the structure constants of corresponding invariant fields are multiplied. For three Neveu-Schwarz primaries or one Neveu-Schwarz and two Ramond primaries we obtain

$$
C_{\left(p_{1} p_{1}^{\prime} q_{1}\right)\left(p_{2} p_{2}^{\prime} q_{2}\right)\left(p_{3} p_{3}^{\prime} q_{3}\right)}^{S(k)}=C_{\left(p_{1}, s_{1}\right)\left(p_{2}, s_{2}\right)\left(p_{3}, s_{3}\right)}^{(k)} C_{\left(s_{1}, q_{1}\right)\left(s_{2}, q_{2}\right)\left(s_{3}, q_{3}\right)}^{(k+1)},
$$

where $s_{i}=\frac{1}{2}\left(p_{i}+q_{i}\right)$ if $p_{i}+q_{i} \in 2 \mathbf{Z}$, and $s_{i}=\frac{1}{2}\left(p_{i}+q_{i} \pm 1\right)$ if $p_{i}+q_{i} \in 2 \mathbf{Z}+1$; $C_{\left(p_{1}, s_{1}\right)\left(p_{2}, s_{2}\right)\left(p_{3}, s_{3}\right)}^{(k)}$ are well known structure constants of minimal model $\mathrm{M}_{k} \cdot{ }^{9}$ For calculations with superpartners it is necessary to take into account the linear combination from Eq. (26).

\section{Acknowlegements}

Author is grateful to S. Kryukov, Ya. P. Pugay, and S. Ye. Parkhomenko for discussion.

\section{References}

1. A. B. Zamolodchikov, Teor. Mat. Fiz. 65 (1985) 347

2. H. Eichenherr, Phys. Lett. B151 (1985) 26

3. M. A. Bershadsky, V. G. Knizhnik and M. G. Teitelman, Phys. Lett. B151 (1985) 31

4. D. Freedan, Z. Qiu and S. Shenker, Phys. Lett. B151 (1985) 37

5. P. Goddard, A. Kent and D. Olive, Commun. Math. Phys. 103 (1986) 105

6. V. G. Knizhnik and A. B. Zamolodchikov, Nucl. Phys. B247 (1984) 83 
7. Vl. S. Dotsenko and V. A. Fateev, Nucl. Phys. B240 [FS12] (1984) 312

8. Vl. S. Dotsenko and V. A. Fateev, Nucl. Phys. B251 [FS13] (1985) 691

9. Vl. S. Dotsenko and V. A. Fateev, Phys. Lett. B154 (1985) 291

10. G. Felder, Nucl. Phys. B317 (1989) 215

11. C. Gomez and G. Sierra, Nucl. Phys. B352 (1991) 791

12. J.-L. Gervais, preprint LPTENS-91/22, hep-th@xxx/9205034, April 1992

13. A. A. Belavin, A. M. Polyakov and A. B. Zamolodchikov, Nucl. Phys. B241 (1984) 333

14. M. Yu. Lashkevich, Int. J. Mod. Phys. A7 (1992) 6623

15. M. Yu. Lashkevich, to be published in Mod. Phys. Lett. A

16. Vl. S. Dotsenko, Adv. Stud. Pure Math. 16 (1988) 123

17. A. B. Zamolodchikov and V. A. Fateev, Sov. Phys. JETP 62 (1985) 215

18. M. Yu. Lashkevich, preprint LANDAU-92-TMP-1, October 1992 